\title{
Experimental Validation of Manipulability Optimization Control of a 7-DoF Serial Manipulator for Robot-assisted Surgery
}

\author{
Hang Su • Andrea Danioni · Robert Mihai \\ Mira • Matteo Ungari • Xuanyi Zhou • \\ Jiehao Li · Yingbai Hu • Giancarlo \\ Ferrigno • Elena De Momi
}

Received: date / Accepted: date

\begin{abstract}
Purpose Both safety and accuracy are of vital importance for surgical operation procedures. An efficient way to avoid the singularity of the surgical robot concerning safety issues is to maximize its manipulability in robot-assisted surgery. The goal of this work was to validate a dynamic neural network optimization method for manipulability optimization control of a 7-DoF robot in a surgical operation. Methods Three different paths, a circle, a sinusoid, and a spiral were chosen to simulate typical surgical tasks. The dynamic neural network-based manipulability optimization control was implemented on a 7-DoF robot manipulator. During the surgical operation procedures, the manipulability of the robot manipulator and the accuracy of the surgical operation are recorded for performance validation. Results By comparison, the dynamic neural network-based manipulability optimization control achieved optimized manipulability but with a loss of the accuracy of trajectory tracking (the global error was $1 \mathrm{~mm}$ compare to the 0.5 $\mathrm{mm}$ error of non-optimized method). Conclusions The method validated in this
\end{abstract}

H. Su, A. Danioni, R. M. Mira, M. Ungari, G. Ferrigno and E. De Momi

Department of Electronics, Information and Bioengineering (DEIB), Politecnico di Milano

Piazza Leonardo da Vinci, 32, 20133 Milano (MI), Italy

E-mail: hang.su@polimi.it

X. Zhou

State Key Laboratory of High Performance Complicated, Central South University

Changsha, 410083, China

J. LI

State Key Laboratory of Intelligent Control and Decision of Complex Systems, Beijing Institute of Technology

Beijing, 100081, China

Correspondence to: $\mathrm{Y}$. Hu

Department of Informatics, Technical University of Munich

Munich, 85748, Germany

E-mail: yingbai.hu@tum.de

Yingbai $\mathrm{Hu}$ would like to thank the support from the Zhejiang Lab's International Talent Fund for Young Professionals. 
work achieved optimized manipulability with a loss of error. Future works should be introduced to improve the accuracy of the surgical operation.

Keywords Trajectory tracking $\cdot$ manipulability $\cdot$ accuracy $\cdot$ redundant robot · robot-assisted surgery

\section{Introduction}

Safety and accuracy represent two key issues in computer-assisted surgery. In the past decades, several studies have been conducted in computer-assisted surgeries to utilize surgical robots performing surgical tasks, driven by the advantages provided by these techniques. For instance, it can achieve higher accuracy in tracking previously planned trajectories [1] without consideration of surgeon fatigue. Moreover, robot-assisted surgery often leads to several benefits, also to the patient. For instance, these are fewer complications as surgical site infections, less pain and blood loss, lower risk of the need for blood transfusion, quicker and easier recovery, smaller and less noticeable scars [2, 3. For the above-mentioned benefits robotic tools assisted surgery is already practiced in general surgery [4] and for several procedures, including for instance mitral valve repair/replacement [5], tumor tissue excision [6, 7], cystectomy [8] and also cosmetic surgery [9].

Especially, redundant robot manipulators attracted many research interests in the past decades, due to their higher number DoFs in the joint space can be utilized to achieve additional tasks simultaneously. Thanks to the redundancy provided by the robot kinematic structure, various research works have been introduced to fascinate the robotic application in surgical robots, including providing the flexibility of workspace [10, 11, maintaining the Remote Center of Motion 12 . and achieving human-like arm pose [13, 14.

Furthermore, it has been proven that their higher number DoFs in the joint space than in the workspace can also be utilized to guarantee or increase flexibility for complicated tasks by optimizing its manipulability 15. An excellent movement ability and the possibility to reach difficult points using the surgical tip can be achieved with the optimization strategy [16, 17]. However, both safe operation environment and a high degree of accuracy must be guaranteed even in unexpected conditions, for example, undesired human-robot interactions [18. For this reason, in addition to the common risks associated with surgical operations, some engineering issues must be addressed. One of the most relevant problems regards kinematic singularity configurations. In these states, the Jacobian matrix loses its rank, the structure loses mobility, and it is impossible to have arbitrary motor laws at the end-effector [19. Moreover, getting close to singularity points, small velocities in the operational space may lead to high velocities in the joint space [20. Hence, it will affect the end-effector moves in specific directions, joint velocities, and accelerations. Also, the large joint velocities may be needed to perform even small displacements or velocities of the end-effector. This behavior of the manipulator is undesirable; it can damage the robot itself, and the sudden joint reconfigurations are extremely dangerous in a surgical environment [21]. Therefore, maximizing the manipulator's performance ability is an efficient way to solve the singularity problems. There are numerous performance ability indexes of robots based on their kinematic properties [22, 23, 24]. Most of the indexes were inspired 
by the manipulability 25]. Manipulability, the most popular of these indexes, will be considered as a major performance index in this paper. The manipulability is the overall manipulator performance of linear/angular velocities at each joint and the end-effector given a certain conguration [26, and it's defined as follow:

$$
\mu=\sqrt{\operatorname{det}\left(J * J^{T}\right)}
$$

In conclusion, the research on manipulability optimization methods plays a crucial role in safety issues in robotic-assisted surgical tasks. Hence, this paper presents an experimental validation of the manipulability optimization control of a 7-DoF Serial robot for robot-assisted surgery to verify its efficiency in terms of the manipulability and the accuracy of the surgical tip.

In this paper, optimization methods that have already been carried out in the literature will be considered, highlighting their pros and cons. Afterward, the methods are applied to a 7-DoFs serial robot performing different trajectories and undergo some possible surgical tasks. In addition, the results are compared and analyzed in order to understand the actual effectiveness of the methods when generalized to different shapes and dimensions trajectories in the surgical field.

The main contributions are summarized as follows:

- In order to effectively avoid the singularity of the surgical robot, dynamic neural network optimization for manipulability optimization control of a 7-DoF robot in surgical operation is considered to the robot-assisted surgery.

- Three experimental paths, including a circle, a sinusoid and a spiral are implemented to achieve the optimization control.

- Some simulation and experiments are carried out to evaluate the accuracy and safety of the proposed method.

\section{State of the art}

Several studies on maximizing redundant robots' manipulability have been investigated in the literature. A correct formulation of both the problem and the function to be optimized is needed.

The first quantitative manipulability measure at the joint velocity level for redundant manipulators was given by T. Yoshikawa. Thus, the singularity of redundant manipulators can be determined by the method of pseudoinverse formulation combined with the manipulability measure. Carlos Garriz proposed a Kalman filter method to optimize the manipulability in a six DOFs manipulator [27]. It is easy to implement, and the states of calculation are based only on previous states. Nevertheless, this method may converge to local solutions [28].

Recently, the optimization method can converge to a global solution, which seems to be the most effective one [29]. It reformulates the redundancy resolution problem with optimal manipulability considered into a constrained quadratic programming problem. However, the solution to the formulated optimization problem cannot be obtained directly. A Neural Network (NN) was an information processing paradigm composed of many highly interconnected processing elements (neurons) working in unison to solve specific problems with adaptive learning and operating in real-time 30 31. The use of DNN for the resolution of redundancy is widely documented in the literature, and different techniques were performed. 
Some of them were in controlling a 6-DoFs robot (PUMA 560) upon a circular trajectory and obtaining in their simulation a (40\%) increase on average on the manipulability value concerning the other methods. Though this method is active, and the work has some limitations considered in this paper. Firstly, the computer simulations had been performed only via self-motion and on a general circular trajectory. Moreover, the method had been applied just on a 6-DoFs manipulator (PUMA 560). The aim of the project presented in this report is indeed to apply the MATLAB code to a 7-DoFs robotic arm (KUKA), adding one additional DoF. Furthermore, the effectiveness of this method was evaluated in different shaped and sized trajectories, simulating a possible use in surgical tasks. It is pivotal to test the algorithm in an environment that is as similar as possible to the one in which the robot is going to work and to choose proper path shapes to mimic possible realistic tasks. However, the complexity of a surgical scenario and the number of different variables and constraints make it hard to satisfy these requirements. In this work, we chose three different surgical tasks, that are two kind of incision and a suture pattern. Then we provided a simplified model of them with the following shapes: a circle, a sinusoid, and a spiral. All these operations are currently performed manually by the surgeon and never by a single-arm autonomous robot. Even though the chosen tasks are quite easy to perform by hand, introducing a redundant surgical robot could significantly increase stability and precision. It leads to a more controlled movement, ensuring a quicker recovery for the patient, less pain, and smaller post-operative scars. It follows a detailed description of the trajectories chosen for the validation of the DNN method.

- Circle. A circle-shaped cutting is quite common, and it can be related to several kinds of surgery, e.g., circular incisions in aesthetic and plastic surgery such as mastectomy or cutting for tumor excision (e.g., craniotomy, mastectomy, kidney tumor removal) [32, [33, [11. In this report, a regular circular pattern, the simplest model for these kinds of cut, with a $2 \mathrm{~cm}$ radius (size consistent with the average areola dimension and a possible tumor mass) was presented. The real shape can change a lot from patient to patient. For instance, for mastectomy and breast reconstruction, the surgeon can choose several incisions of different shapes, most of which based on a circular geometry (as periareolar or elliptical incisions) 33. Additionally, the tumor excision, which can interest both hard or soft tissues, masses of different sizes and shape may involve a round incision to remove cancer. The presence of robotic assistance may allow the surgeon to perform these tasks with enhanced dexterity, stability, and precision, reducing the presence of scars and leading the patient to a faster recovery. For example, it has been shown that semi-autonomous electrosurgery for tumor resection performed by a robotic arm is more accurate and stable than the human hand. It can also avoid complications due to long operations and visualization.

- Sinusoid. A prime example of a sinusoidal trajectory used in the surgical environment is the coronal incision, which is typically sinusoidal (Fig. 2) or sawtooth-shaped. As the name suggests, this incision is done along the coronal plane of the human body, running the head of the patient from one ear to the other along the cranial circumference. In this report, a $25 \mathrm{~cm}$ long sinusoid has been chosen in order to be sensible for the head dimension. It has undergone many modifications during the years in order to optimize the aesthetic appear- 

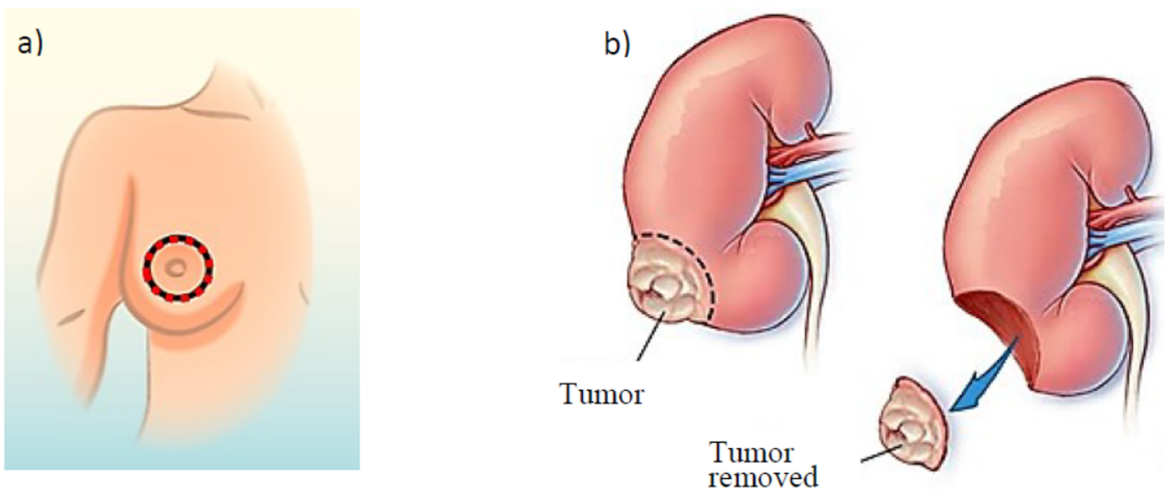

Fig. 1: a) periareolar incision trace for breast surgery; b) round incision for kidney tumor removal.
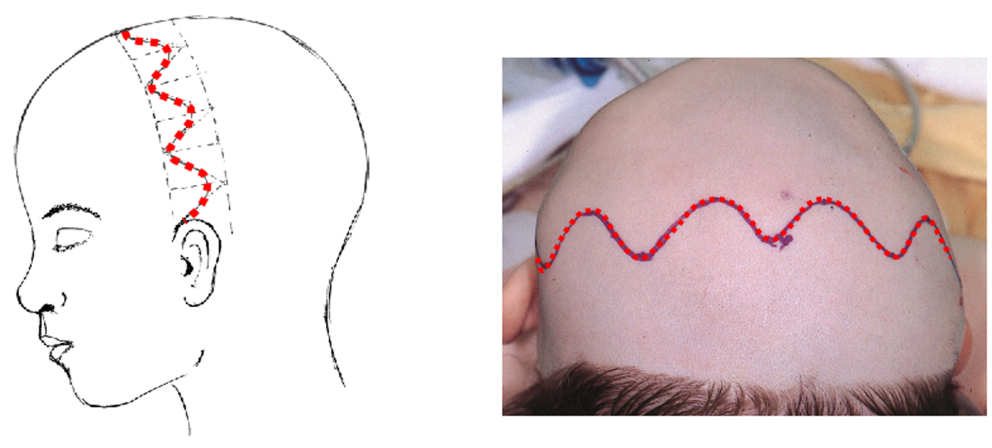

Fig. 2: Sinusoidal shaped coronal incision trace. The shape and the dimensions of the incision are usually chosen in order to hide as much as possible the postoperative scars.

ance of the scar. It provides excellent exposure to the cranial vault and the upper part of the facia bone structure allowing neurosurgical access, craniofacial osteotomies, repair of facial fractures, calvarial bone grafting, and cosmetic procedures [34], 35]. Some disadvantages of this kind of incision, as the presence of big scars, the long operation time, the risk of infections and hemorrhage, the palsy of the facial nerve [36, may be reduced thanks to the usage of robotic assistance. Thus, no application of robotic tools for the execution of this task is present in literature yet.

- Spiral. This is one of the typical suture patterns [37]. In some studies, it is pointed out how the automatization of the suturing task may reduce both the time and difficulty of completing a suture and free the surgeon from repetitive subtasks. Moreover, preplanning the autonomous motion would allow the 


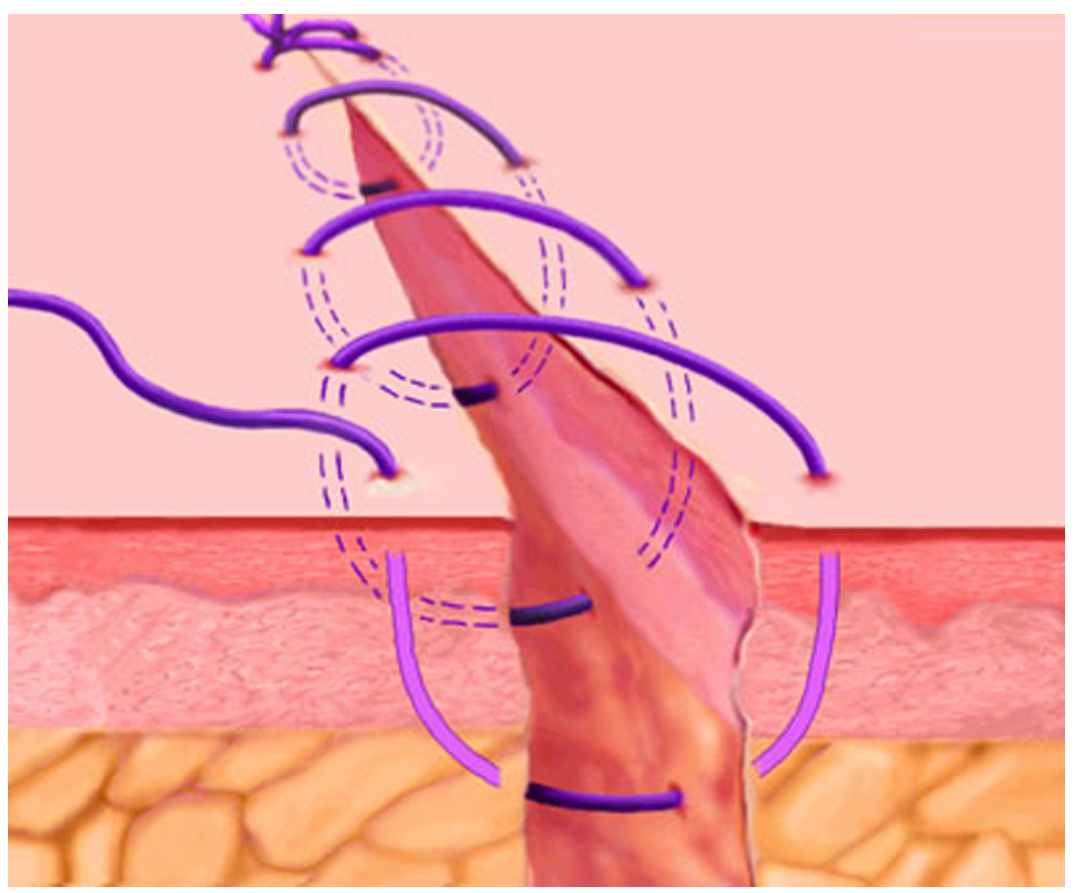

Fig. 3: Simple continuous suture simulation, pointing out the typical spiral pattern.

robot to minimize any tissue trauma that might occur during suturing. In this report, a $5 \mathrm{~cm}$ length spiral with a 2 centimeters diameter will be used. The characteristic dimensions of the suture can obviously vary a lot, depending on the type of injury. However, for this kind of task, we must consider a big simplification: a single robotic arm cannot continuously perform this task, but the end-effector must periodically release and re-grab the needle, in the way to insert and pull it properly.

\section{Materials and Methods}

We first begin choosing a set of different shape trajectories with proper dimensions in order to simulate some examples of surgical tasks. The chosen paths were a circle, a sinusoid and a spiral, for the reasons described in the state of art chapter.

The measure chosen for these shapes, according to real surgery scenarios, are enumerated in Table 1 . The use of different paths with different dimensions aims to be further proof of the method's validity.

\subsection{Code Implementation}

The forward kinematics for a redundant manipulator provides a nonlinear mapping from the joint space to the Cartesian space. Thus, it is difficult to solve directly 
Table 1: Main dimensions of the chosen trajectory patterns. $R=$ Radius; $A=$ Amplitude; L = Length.

\begin{tabular}{c|c}
\hline Pattern & Dimensions \\
\hline Circle & $R=2 \mathrm{~cm}$ \\
Sinusoid & $A=1 \mathrm{~cm} ; L=25 \mathrm{~cm}$ \\
Spiral & $R=1 \mathrm{~cm} ; L=5 \mathrm{~cm}$ \\
\hline
\end{tabular}

the redundancy problem at the angle level [38] 39]. Due to these reasons, we implemented our code, firstly converting the problem into a problem at the velocity level, applying the pseudoinverse formulation for obtaining the general solution at the joint velocity level, that is, the desired velocity of the manipulator's joints at each step.

The code implemented in this work defines the whole trajectory and divides it in an arbitrary number of steps $\mathrm{N}$ (we have chosen $\mathrm{N}=1000$ for our simulations). Starting from an arbitrary joint configuration, the desired Cartesian position is then defined. V, which is cartesian velocity, is computed (2). Moreover, the Jacobian matrix, that is a function of the joint configuration q updated in (3). Through both the pseudoinverse of the Jacobian matrix and the cartesian velocity, it is easy to compute the joint velocity (4) and derive then the desired joint position $q_{\text {des }}$ (5). It is possible then to define the next robot's Cartesian position for each step using the forward kinematics on the obtained joint configuration $q_{\text {des }}$. Performing a "for cycle" over these passages, it is possible to obtain the whole trajectory.

We calculated the manipulability value and the error as the difference between the desired pose and the actual one for each joint configuration.

$$
\begin{gathered}
v=\frac{d r}{d t} \\
J_{r}(q)=\frac{\delta f_{r}(q)}{\delta q} \\
\dot{q}=\operatorname{pinv}\left(J_{r}(q)\right) * \dot{r} \\
q-d e s=q+\dot{q} * t
\end{gathered}
$$
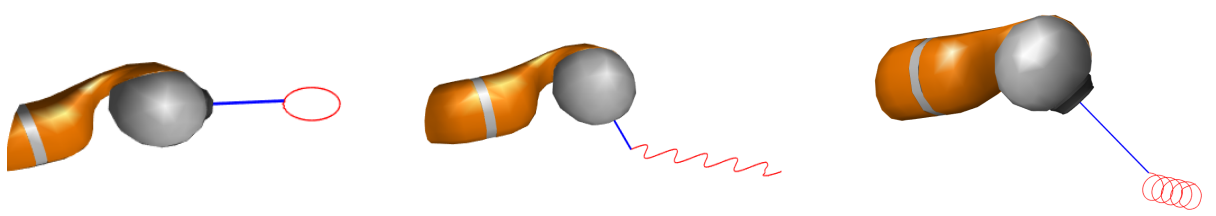

Fig. 4: Graphical representation of the obtained trajectory performed by the robotic arm. From the left: circular trajectory, sinusoidal trajectory, spiral trajectory. 
Table 2: List of parameters and operators used in the set of differential equations (6), (7), (8), (9), explained with their meaning.

\begin{tabular}{|c|c|c|c|}
\hline VARIABLE & \multicolumn{3}{|c|}{ MEANING } \\
\hline$\varepsilon$ & \multicolumn{3}{|c|}{ Positive scaling factor } \\
\hline$\omega=\dot{\theta}$ & \multirow{2}{*}{\multicolumn{3}{|c|}{$\begin{array}{c}\text { Joint Velocity } \\
\partial J / \partial \theta_{i}\end{array}$}} \\
\hline$H_{i}$ & \multirow{2}{*}{\multicolumn{3}{|c|}{$\begin{array}{c}\partial J / \partial \theta_{i} \\
\text { Projection Operator }\end{array}$}} \\
\hline$P_{\Omega}$ & & & \\
\hline & \multirow{3}{*}{\multicolumn{3}{|c|}{$\begin{array}{c}\varphi \in R^{m 2}, \text { is an stimation of } \operatorname{vec}\left(J\left(J_{T}\right)^{(-1)}\right) \\
\text { State Variables } \\
\text { Positive constants }\end{array}$}} \\
\hline & & & \\
\hline$c 0, c 1, c 2, c 3$ & & & \\
\hline OPERATOR & \multicolumn{3}{|c|}{ MEANING } \\
\hline 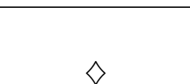 & $J \diamond\left(H_{1}, H_{2}, \ldots, H_{k}\right)=$ & $\begin{array}{l}\operatorname{vec}^{T}\left(H_{1}^{T}\right) \\
\operatorname{vec}^{T}\left(H_{2}^{T}\right)\end{array}$ & $\left(I_{m} \otimes J^{T}\right)$ \\
\hline$\otimes$ & Kronect & $\begin{array}{l}\operatorname{vec}^{T}\left(H_{k}^{T}\right) \\
\text { ker Product }\end{array}$ & \\
\hline
\end{tabular}

\subsection{Application of the Optimization Control}

Thereafter, we modified the process introducing the optimizing part, keeping as a reference to the method developed in 40. Since this work focuses on the validation of the manipulability optimization proposed, it won't describe how their Neural Network is derived.

First, the above-mentioned NN has been implemented, represented by the following set of differential equations:

$$
\begin{gathered}
\varepsilon \dot{\omega}=-\omega+P_{\Omega}\left[c_{3}\left(J \diamond\left\{H_{1}, H_{2}, \ldots, H_{k}\right\}\right) \varphi\right. \\
\left.-c_{0} J^{T}\left(J \omega-v_{d}\right)+\left(1-c_{2}\right) \omega-J^{T} \lambda\right] \\
\varepsilon \dot{\varphi}=-c_{3}\left(J \diamond\left\{H_{1}, H_{2}, \ldots, H_{k}\right\}\right)^{T} \omega+\left(I_{m} \otimes J J^{T}\right) \sigma \\
+c_{1}\left(\left(I_{m} \otimes J J^{T}\right)\left[\left(I_{m} \otimes J J^{T}\right) \varphi-\operatorname{vec}\left(I_{m}\right)\right]\right) \\
\varepsilon \dot{\lambda}=J \omega-v_{d} \\
\varepsilon \dot{\sigma}=\operatorname{vec}\left(I_{m}\right)-\left(I_{m} \otimes J J^{T}\right) \varphi
\end{gathered}
$$

Given a certain Cartesian velocity $v_{d}$, it solves the redundancy problem and outputs the correspondent joint acceleration such that the maximum value of manipulability is reached. From the obtained joint acceleration, we derive the desired joint position, and the robot poses then. The described set of differential equations controls the variation of three-time dependent parameters and of the joint space acceleration. It resolves the redundancy problem relying on several tuning parameters. Further, the optimization is obtained by tuning the parameters such that the maximum value of $\mu$ is reached, and an acceptable error is developed. The parameters and the operators used in the previous set of differential equations are explained in the following table (Table 2).

The original code in [32] was developed for a 6-DoFs (PUMA 560 manipulator), while the KUKA manipulator considered in this paper holds 7-DoFs. Therefore, some modifications have been performed with respect to the original algorithm, in order to consider the added DoF. The kinematic model of 7 DoFs LightWeight 
Table 3: D-H parameters of the KUKA robot. $\theta$ is the angle between link axes (variable for revolute joint); $\mathrm{d}$ is the displacement between link axes (variable for prismatic joint); $a$ is the displacement between joint axes (common normal); $\alpha$ is the twist angle between joint axes.

\begin{tabular}{c|c|c|c|c}
\hline Link & $\theta$ & $d$ & $a$ & $\alpha$ \\
\hline 1 & $\theta_{1}$ & 0.31 & 0 & 1.5708 \\
2 & $\theta_{2}$ & 02 & 0 & -1.5708 \\
3 & $\theta_{3}$ & 0.4 & 0 & -1.5708 \\
4 & $\theta_{4}$ & 0 & 0 & 1.5708 \\
5 & $\theta_{5}$ & 0.39 & 0 & 1.5708 \\
6 & $\theta_{6}$ & 0 & 0 & -1.5708 \\
7 & $\theta_{7}$ & 0.078 & 0 & 0 \\
\hline
\end{tabular}

robotic arm (LWR4+, KUKA, GERMANY) considered in this paper is defined using Denavit-Hartenberg $(\mathrm{D}-\mathrm{H})$ notation, and the corresponding $\mathrm{D}-\mathrm{H}$ parameters are listed in Table 3.

The position accuracy of surgery robots is a critical requirement for patient safety since it affects the surgical operation directly [41. In order to assess the validity of the method and the manipulability improvement effectiveness, it is required that the desired cartesian trajectory is accurately followed by the robot, with small and comparable errors for both the methods. The trajectory tracking error can affect both the surgery result and the manipulability value. The obtained error values are described in the next section, Results.

Because the manipulability index is determined only by the kinematic placement, there is no difference to validate it with a real robot or with simulation. To ease the analysis, we performed the validation using simulation. All the simulations for validation of the method described in this report have been performed on MATLAB. In order to evaluate the results (presented in the next section), we created both standard line plots and boxplots for the manipulability value. For each of the three different trajectory paths, the simulation was performed four times (using four different starting configurations in order to make the method as general as possible).

\section{Results and Discussions}

The value of manipulability is the main parameter measure in order to verify the effectiveness of the described method. Although the range of increments in manipulability cannot be constant, it strongly depends on the task and the starting position. It holds that for every trajectory path and each starting configuration considered in this project, an increase in manipulability can be measured, with an improvement step by step. In the following plots, the comparison between the manipulability values is reported for the three different tasks that the robot performed in Fig. 5 .

The following picture shown in Fig. 6 represents the manipulability comparison between the four different starting positions that we randomly chose to make a general validation of the method. We can assert that an improvement of the manipulability is always attained. However, the effect of improvement is highly 
(a) Circle Manipulability Comparison

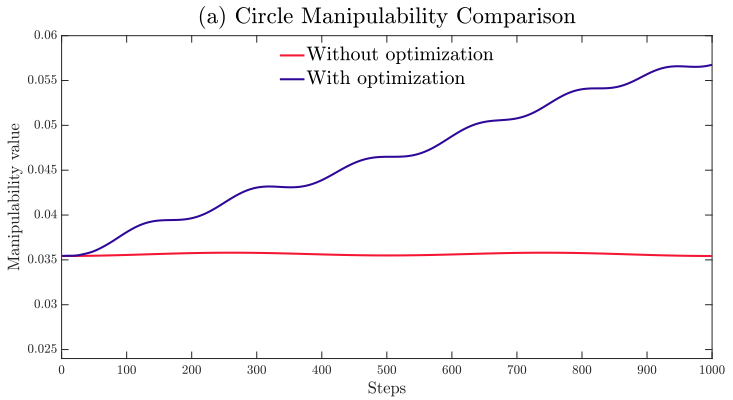

(b) Sine Manipulability Comparison

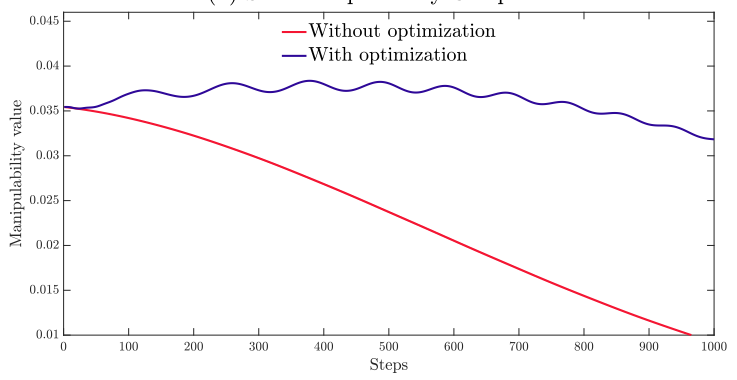

(c) Spiral Manipulability Comparison

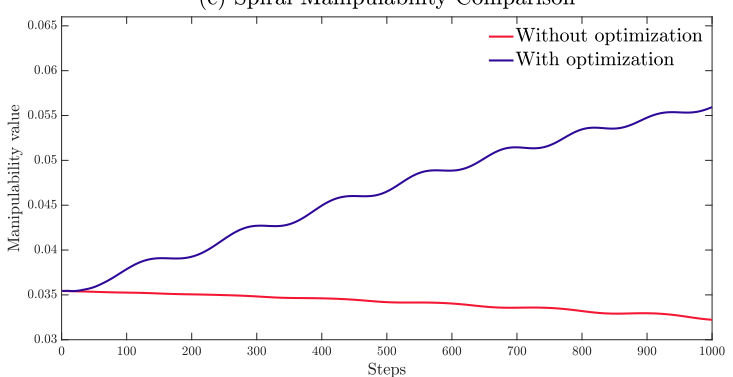

Fig. 5: Plots that represent the manipulability values comparison, with the optimization method (blue) and without it (red). In order they are referred to the circular, sinusoidal and spiral path, all for the same starting configuration.

relevant to the starting configuration of the robot, and the manipulability improved slightly. The reported plots refer to the spiral trajectory taken as an example.

In order to visually compare the median values of the manipulability over the whole 1000 steps, we decided to represent the boxplots corresponding to the diagrams already shown. Some examples concerning the spiral trajectory are shown in Fig. 7.

As shown in this section, the presented method proved effective in all the performed simulations, increasing the overall manipulability with respect to the pseudoinverse method. Nevertheless, the manipulability increase depends on the shape of the trajectory and the number of steps used to segment the path. The obtained results that have been shown before are entirely collected in Table 4 with their numerical values. 

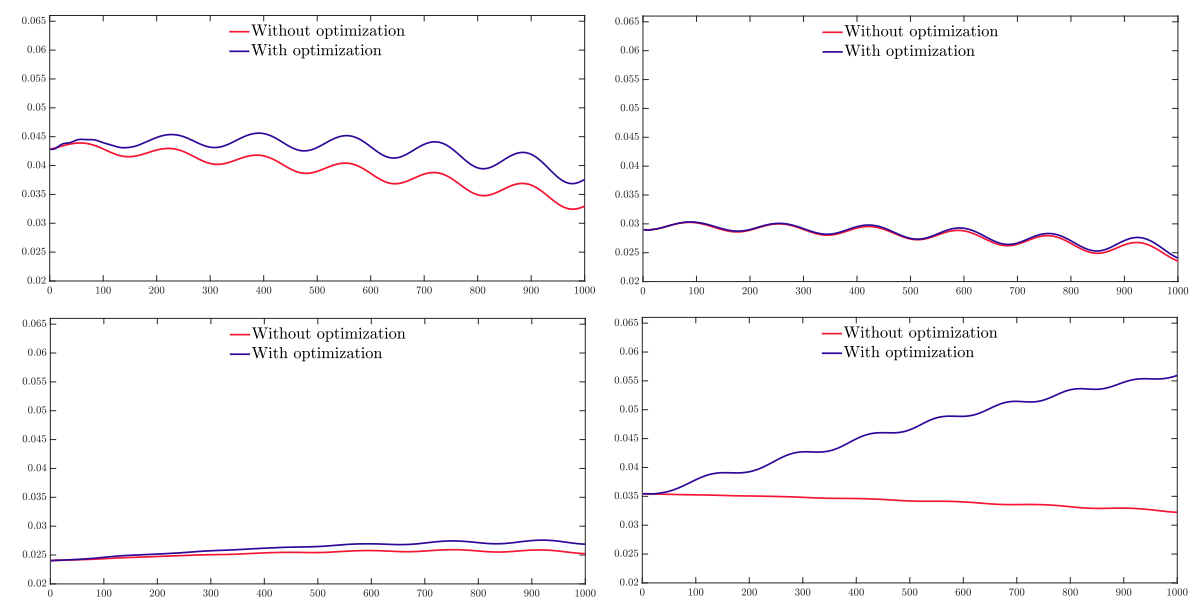

Fig. 6: Manipulability comparison between the four spiral trajectories performed, starting from four different configurations. In each case an increment in the manipulability value is obtained, improving step by step.

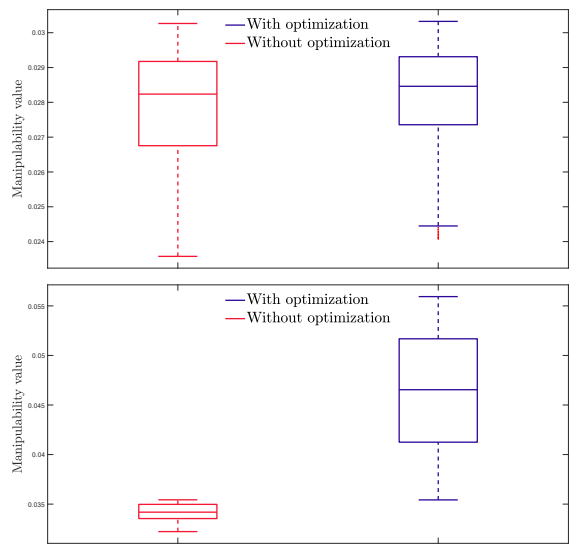

Fig. 7: Boxplots that gives a visual representation of the manipulability median value and the interval of values assumed on the overall trajectory. The reported plots concern the spiral trajectory starting from two different configurations.

The important result obtained in this project was to make the robot perform the three different trajectories, as mentioned previously accurately. In order to verify that we plotted the desired cartesian trajectory and the real cartesian trajectory in the three directions ( $\mathrm{x}, \mathrm{y}, \mathrm{z})$, displayed in Fig. 8, the global error measured in meters as the difference between the desired and the real trajectory shown in Fig. 9. Further, we made the same evaluation for the trajectories to which the manipulability optimization has been applied. We noticed that the error magnitude is larger than the non-optimized trajectories, especially in the very initial steps. Due to the optimization method adopted in this project, oscillations of some millime- 
Table 4: Manipulability values expressed as an arithmetic average over all the steps in which the trajectory is segmented. The not optimized value, the optimized value, the average increase, and the percentage increase are represented for each trajectory shape and for each starting position considered in this project.

\begin{tabular}{|c|c|c|c|c|}
\hline & \multicolumn{4}{|c|}{ CIRCULAR TRAJECTORY } \\
\hline & q_home_1 & q_home_2 & q_home_3 & q_home_4 \\
\hline $\begin{array}{l}\text { NOT OPTIMIZED } \\
\text { MANIPULABILITY AVERAGE }\end{array}$ & 0,0406 & 0,0282 & 0,0246 & 0,0356 \\
\hline OPTIMIZED & 0.0448 & 0.0287 & 0.0264 & $0 \quad 0466$ \\
\hline MANIPULABILITY AVERAGE & & & & \\
\hline $\begin{array}{l}\text { AVERAGE MANIPULABILITY } \\
\text { INCREASE }\end{array}$ & 0,0042 & 0,0005 & 0,0018 & 0,0110 \\
\hline$A V E R A G E$ INCREASE \% & $10,3 \%$ & $1,8 \%$ & $7,3 \%$ & $30,9 \%$ \\
\hline & \multicolumn{4}{|c|}{ SINUSOIDAL TRAJECTORY } \\
\hline & q_home_1 & q_home_2 & q_home_3 & q_home_4 \\
\hline $\begin{array}{l}\text { NOT OPTIMIZED } \\
\text { MANIPULABILITY AVERAGE }\end{array}$ & 0,0447 & 0,0281 & 0,0222 & 0,0233 \\
\hline $\begin{array}{c}\text { OPTIMIZED } \\
\text { MANIPULABILITY AVERAGE }\end{array}$ & 0,0512 & 0,0303 & 0,0249 & 0,0363 \\
\hline $\begin{array}{l}\text { AVERAGE MANIPULABILITY } \\
\text { INCREASE }\end{array}$ & 0,0065 & 0,0022 & 0,0027 & 0,0130 \\
\hline \multirow[t]{2}{*}{ AVERAGE INCREASE \% } & $14,5 \%$ & $7,8 \%$ & $12,2 \%$ & $55,8 \%$ \\
\hline & \multicolumn{4}{|c|}{ SPIRAL TRAJECTORY } \\
\hline & q_home_1 & q_home_2 & q_home_3 & q_home_4 \\
\hline $\begin{array}{l}\text { NOT OPTIMIZED } \\
\text { MANIPULABILITY AVERAGE }\end{array}$ & 0,0393 & 0,0280 & 0,0253 & 0,0342 \\
\hline $\begin{array}{c}\text { OPTIMIZED } \\
\text { MANIPULABILITY AVERAGE }\end{array}$ & 0,0429 & 0,0282 & 0,0262 & 0,0465 \\
\hline $\begin{array}{l}\text { AVERAGE MANIPULABILITY } \\
\text { INCREASE }\end{array}$ & 0,0036 & 0,0003 & 0,0010 & 0,0123 \\
\hline$A V E R A G E I N C R E A S E \%$ & $9,2 \%$ & $1,1 \%$ & $3,9 \%$ & $36,0 \%$ \\
\hline
\end{tabular}

ters occur in the very initial steps and eliminates step after step. It is possible to appreciate this behavior in Fig. 8

Finally, to validate the effect the optimized method has with the improvement in manipulability, we evaluated the global error. The example plot is shown in Fig. 10, relative to the spiral trajectory, demonstrates the comparability between the errors. The errors of the optimized method are in a higher order of magnitude (around $1 \mathrm{~mm}$ ) than the unoptimized method (around $0.5 \mathrm{~mm}$ ). The numerical values of the errors obtained with and without optimization method are compared in Table 5.

\section{Conclusion and Future Work}

This paper validates a manipulability optimization control of a redundant serial robot for performing surgical tasks. The manipulability can be optimized while its performance is highly dependent on the shape of the trajectory and the starting position. Furthermore, the error of this optimization method is magnified with increasing manipulability. In this research, the method above was applied to three different surgical tasks, and it was, in general, sufficient for each randomly chosen starting configuration. However, the starting configuration and the shape of the 

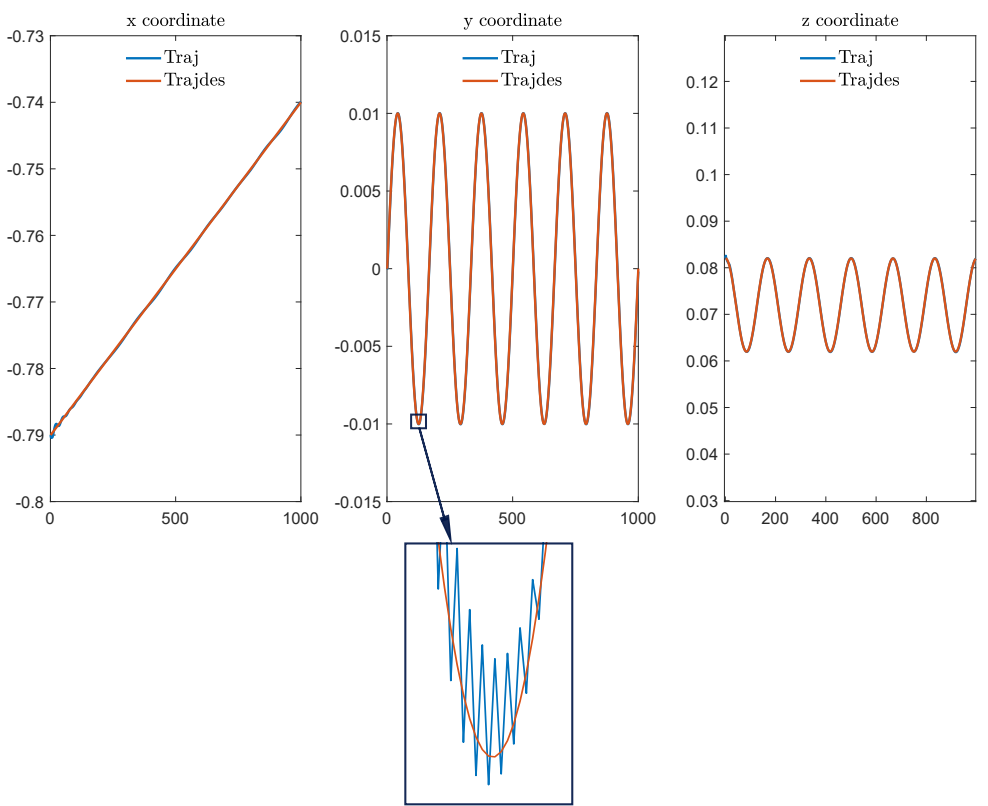

Fig. 8: Comparison between the desired trajectory (red) and the real trajectory (blue) for the sinusoidal path, taken as example. We can notice that the difference between the two trajectory is in the order of tenths of a millimeter. This order of magnitude is similar for all the paths and for all the starting configurations.

Table 5: representation of the root mean square error values for each trajectory shape and for each starting position. All the values are expressed in meter $s * 10^{-4}$. The values are computed as: $R M S=\sqrt{\frac{\sum(\varepsilon)^{2}}{N}}$.

\begin{tabular}{|c|c|c|c|c|}
\hline & \multicolumn{4}{|c|}{ CIRCULAR TRAJECTORY } \\
\hline $\begin{array}{l}\text { ROOT MEAN SQUARE } \\
\text { ERROR }\end{array}$ & q_home_1 & q_home_2 & q_home_3 & q_home_4 \\
\hline Optimization method & 3.992 & 3.876 & 2.433 & 67 \\
\hline Pseudo in & & & & \\
\hline & \multicolumn{4}{|c|}{ SINUSOIDAL TRAJECTORY } \\
\hline $\begin{array}{l}\text { ROOT MEAN SQUARE } \\
\text { ERROR }\end{array}$ & q_home_1 & q_home_2 & q_home_3 & q_home_4 \\
\hline Optimizatio & & & & \\
\hline Pseudo inverse & & 2.062 & & \\
\hline & \multicolumn{4}{|c|}{ SOLENOIDAL TRAJECTORY } \\
\hline $\begin{array}{l}\text { ROOT MEAN SQUARE } \\
\text { ERROR }\end{array}$ & q_home_1 & q_home_2 & q_home_3 & q_home_4 \\
\hline $\begin{array}{l}\text { Optimization method } \\
\text { Pseudo inverse }\end{array}$ & & & $\begin{array}{l}4.389 \\
4.151\end{array}$ & $\begin{array}{l}3.778 \\
3.590\end{array}$ \\
\hline
\end{tabular}

trajectory impact the performance of manipulability. Optimizing the starting configuration and the shape of the trajectory will be part of the future work. While performing the simulations, we realized that the manipulator does not care about possible geometrical constraints of a real surgical scenario, for example, the pres- 


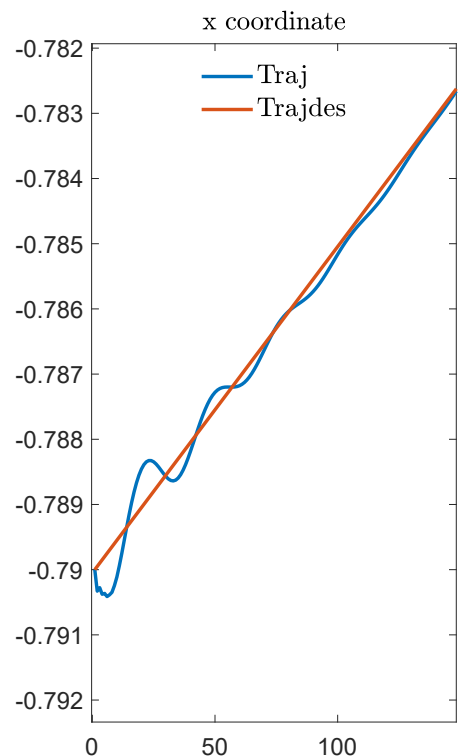

Fig. 9: Comparison between the desired trajectory (red) and the real trajectory (blue) for the sinusoidal path is taken as an example. We can notice that the difference between the two trajectories is in the order of tenths of a millimeter. This order of magnitude is similar for all the paths and for all the starting configurations.

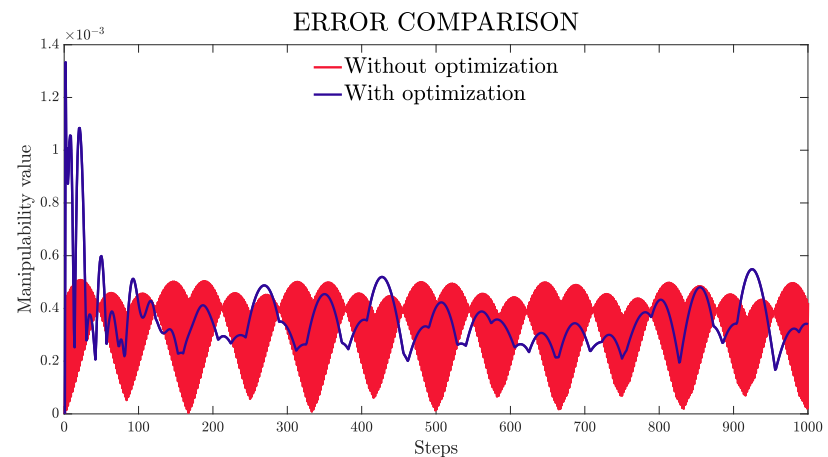

Fig. 10: Error comparison between the same sinusoidal Cartesian trajectory without optimization (red) and with optimization (blue).

ence of the patient or other possible obstacles. Hence, the developed method cannot be applied alone for the execution of surgical tasks. As future work, we suggest implementing an algorithm that combines the manipulability optimization with the real path planning constraints to obtain a manipulator capable of executing the task safely, avoiding any possible obstacle and maximizing the manipulability value at the same time. 


\section{References}

1. Kim H, Rosen J. Predicting redundancy of a 7 dof upper limb exoskeleton toward improved transparency between human and robot Journal of Intelligent and Robotic Systems. 2015;80:99-119.

2. Camarillo D. B, Krummel T. M, Salisbury Jr J. K. Robotic technology in surgery: past, present, and future The American Journal of Surgery. 2004;188:2-15.

3. Li J, Wang J, Peng H, Zhang L, Hu Y, Su H. Neural fuzzy approximation enhanced autonomous tracking control of the wheel-legged robot under uncertain physical interaction Neurocomputing. 2020;410:342-353.

4. Marano A, Priora F, Lenti L. M, Ravazzoni F, Quarati R, Spinoglio G. Application of fluorescence in robotic general surgery: review of the literature and state of the art World journal of surgery. 2013;37:2800-2811.

5. Gillinov A. M, Suri R, Mick S, Mihaljevic T. Robotic mitral valve surgery: current limitations and future directions Annals of Cardiothoracic Surgery. 2016;5:573.

6. Yang H. C, Coyan G, Vercauteren M, Reddy N, Luketich J. D, Sarkaria I. S. Robot-assisted en bloc anterior mediastinal mass excision with pericardium and adjacent lung for locally advanced thymic carcinoma Journal of Visualized Surgery. ;4:115-115.

7. Potretzke A. M, Weaver J, Benway B. M. Review of robot-assisted partial nephrectomy in modern practice Journal of Kidney Cancer \& Vhl. 2015;2:3044.

8. Tyritzis S. I, Collins J, Wiklund N. P. The current status of robot-assisted cystectomy Indian Journal of Urology Iju Journal of the Urological Society of India. 2018;34:101-109.

9. Ahn S. J, Song S. Y, Park H. S, et al. Early experiences with robot-assisted prosthetic breast reconstruction Archives of plastic surgery. 2019;46:79.

10. Su H, Qi W, Yang C, Sandoval J, Ferrigno G, De Momi E. Deep neural network approach in robot tool dynamics identification for bilateral teleoperation IEEE Robotics and Automation Letters. 2020.

11. Qi W, Zong G, Karimi H. R. Sliding mode control for nonlinear stochastic semi-Markov switching systems with application to SRMM IEEE Transactions on Industrial Electronics. 2019;67:3955-3966.

12. Su H, Yang C, Ferrigno G, De Momi E. Improved Human-Robot Collaborative Control of Redundant Robot for Teleoperated Minimally Invasive Surgery IEEE Robotics and Automation Letters. 2019;4:1447-1453.

13. Su H, Enayati N, Vantadori L, Spinoglio A, Ferrigno G, De Momi E. Online human-like redundancy optimization for tele-operated anthropomorphic manipulators International Journal of Advanced Robotic Systems. 2018;15:1729881418814695.

14. Su H, Qi W, Yang C, Aliverti A, Ferrigno G, De Momi E. Deep Neural Network Approach in Human-like Redundancy Optimization for Anthropomorphic Manipulators IEEE Access. 2019.

15. Zhang Z, Zheng L, Chen Z, Kong L, Karimi H. R. Mutual-Collision-Avoidance Scheme Synthesized by Neural Networks for Dual Redundant Robot Manipulators Executing Cooperative Tasks IEEE Transactions on Neural Networks and Learning Systems. 2020. 
16. Chen Y, Kwok K.-W, Tse Z. T. H. An MR-conditional high-torque pneumatic stepper motor for MRI-guided and robot-assisted intervention Annals of biomedical engineering. 2014;42:1823-1833.

17. Su H, Hu Y, Karimi H. R, Knoll A, Momi E. D. Improved recurrent neural network-based manipulator control with remote center of motion constraints: Experimental results Neural Networks. 2020.

18. Li J, Wang J, Wang S, et al. Parallel structure of six wheel-legged robot trajectory tracking control with heavy payload under uncertain physical interaction Assembly Automation. 2020;40:675-687.

19. Pavri S. N, Arnaud E, Renier D, Persing J. A. The Posterior Coronal Incision Journal of Craniofacial Surgery. 2015;26:243-244.

20. Krishnan S, Garg A, Patil S, et al. Transition state clustering: Unsupervised surgical trajectory segmentation for robot learning The International Journal of Robotics Research. 2017;36:1595-1618.

21. Zhou X, Qi W, Ovur S. E, et al. A novel muscle-computer interface for hand gesture recognition using depth vision Journal of Ambient Intelligence and Humanized Computing. 2020:1-12.

22. Cardou P, Bouchard S, Gosselin C. Kinematic-Sensitivity Indices for Dimensionally Nonhomogeneous Jacobian Matrices IEEE Transactions on Robotics. 2010;26:166-173.

23. Li J, Wang J, Wang S, et al. Neural Approximation-based Model Predictive Tracking Control of Non-holonomic Wheel-legged Robots International Journal of Control, Automation and Systems. 2020:1-10.

24. Mansouri I, Ouali M. The power manipulability-A new homogeneous performance index of robot manipulators Robotics and Computer-Integrated Manufacturing. 2011;27:434-449.

25. Li S, He J, Li Y, Rafique M. U. Distributed recurrent neural networks for cooperative control of manipulators: A game-theoretic perspective IEEE transactions on neural networks and learning systems. 2016;28:415-426.

26. Prattichizzo D, Malvezzi M, Gabiccini M, Bicchi A. On the manipulability ellipsoids of underactuated robotic hands with compliance Robotics and Autonomous Systems. 2012;60:337-346.

27. Garriz C, Domingo R. Development of Trajectories Through the Kalman Algorithm and Application to an Industrial Robot in the Automotive Industry IEEE Access. 2019;7:23570-23578.

28. Koç O, Maeda G, Peters J. Online optimal trajectory generation for robot table tennis Robotics and Autonomous Systems. 2018;105:121-137.

29. Kwok K.-W, Chen Y, Chau T. C, et al. MRI-based visual and haptic catheter feedback: simulating a novel system's contribution to efficient and safe MRIguided cardiac electrophysiology procedures Journal of Cardiovascular Magnetic Resonance. 2014;16:1-3.

30. Momi E. D, Kranendonk L, Valenti M, Enayati N, Ferrigno G. A Neural Network-Based Approach for Trajectory Planning in Robot-Human Handover Tasks Frontiers in Robotics and AI. 2016;3.

31. $\mathrm{Hu} \mathrm{Y,} \mathrm{Wu} \mathrm{X,} \mathrm{Geng} \mathrm{P,} \mathrm{Li} \mathrm{Z.} \mathrm{Evolution} \mathrm{strategies} \mathrm{learning} \mathrm{with} \mathrm{variable}$ impedance control for grasping under uncertainty IEEE Transactions on Industrial Electronics. 2018;66:7788-7799.

32. Burke J. F, Sudhakar V, Braunstein S, McDermott M. In Situ Cranioplasty for Renal Cell Skull Metastasis Cureus. 2019;11. 
33. Kinoshita S, Kyoda S, Hirano A, et al. Clinical comparison of four types of skin incisions for skin-sparing mastectomy and immediate breast reconstruction Surgery today. 2014;44:1470-1475.

34. Fox A. J, Tatum S. A. The coronal incision: sinusoidal, sawtooth, and postauricular techniques Archives of facial plastic surgery. 2003;5:259-262.

35. Pavri S. N, Arnaud E, Renier D, Persing J. A. The Posterior Coronal Incision Journal of Craniofacial Surgery. 2015;26:243-244.

36. Qing-Bin Z, Yao-Jun D, Zu-Bing L, Ji-Hong Z. Coronal incision for treating zygomatic complex fractures Journal of Cranio-Maxillofacial Surgery. 2006;34:182-185.

37. Krishnan S, Garg A, Patil S, et al. Transition state clustering: Unsupervised surgical trajectory segmentation for robot learning The International Journal of Robotics Research. 2017;36:1595-1618.

38. Sagitov A, Tsoy T, Li H, Magid E. Automated open wound suturing: detection and planning algorithm Journal of Robotics, Networking and Artificial Life. 2018;5:144-148.

39. Chang N. C, Jung H, Son J, Kim K. G. A modular control scheme for hyper-redundant robots International Journal of Advanced Robotic Systems. 2015;12:1.

40. Jin L, Li S, La H. M, Luo X. Manipulability optimization of redundant manipulators using dynamic neural networks IEEE Transactions on Industrial Electronics. 2017;64:4710-4720.

41. Ji D, Kang T. H, Shim S, Lee S, Hong J. Wire-driven flexible manipulator with constrained spherical joints for minimally invasive surgery International Journal of Computer Assisted Radiology and Surgery. 2019. 